\title{
Article \\ Active Video Game Program for People with Type 2 Diabetes- a Pilot Study
}

\author{
Han-Hung Huang ${ }^{1, *}$, Brianna Gathright ${ }^{1}$, Rachel Holik ${ }^{1}$, Hannah Iverson ${ }^{1}$, Emily Saville ${ }^{1}$ \\ and Drew A. Curtis ${ }^{2}$ (D) \\ 1 Department of Physical Therapy, Angelo State University, Member, Texas Tech University System, \\ San Angelo, TX 76904, USA; bgathright@angelo.edu (B.G.); rachelizabeth.holik@gmail.com (R.H.); \\ hlkiverson@gmail.com (H.I.); emily_saville@yahoo.com (E.S.) \\ 2 Department of Psychology, Angelo State University, Member, Texas Tech University System, \\ San Angelo, TX 76904, USA; drew.curtis@angelo.edu \\ * Correspondence: floyd.huang@angelo.edu; Tel.: +1-325-942-2627
}

check for updates

Citation: Huang, H.-H.;

Gathright, B.; Holik, R.; Iverson, H.; Saville, E.; Curtis, D.A. Active Video Game Program for People with Type 2 Diabetes- a Pilot Study. Appl. Sci. 2021, 11, 11046. https://doi.org/ 10.3390/app112211046

Academic Editor: Shin-Da Lee

Received: 30 September 2021

Accepted: 8 November 2021

Published: 22 November 2021

Publisher's Note: MDPI stays neutral with regard to jurisdictional claims in published maps and institutional affiliations.

Copyright: (c) 2021 by the authors. Licensee MDPI, Basel, Switzerland. This article is an open access article distributed under the terms and conditions of the Creative Commons Attribution (CC BY) license (https:// creativecommons.org/licenses/by/ $4.0 /)$.
Featured Application: Rehabilitation for People with Type 2 Diabetes.

Abstract: The objective of this study was to assess the effects of an active video game (AVG) program in physiological parameters, fitness levels, motivation to physical activity, and quality of life on people with type 2 diabetes (T2D). Eight T2D patients participated in the 8-week AVG program using Xbox Kinect and/or Nintendo Wii. Heart rate (HR) and rating of perceived exertion (RPE) were monitored during exercise. Physiological and fitness assessment included hemoglobin A1C (HbA1c), HR, blood pressure (BP), body mass index, body composition, aerobic and muscular endurance, muscular strength, and flexibility. Psychological outcome measures included the Physical Activity Measure-Revised (MPAM-R), Situational Motivation Scale (SIMS), and World Health Organization Quality of Life (WHOQOL)-BREF. Participants demonstrated a moderate level of exercise intensity during AVG based on average HR and RPE. HbA1c, BP, and lower extremity muscle strength were improved, but were not statistically significant. The changes on psychological assessment were not significant. However, most participants demonstrated positive behavior and attitudes toward health and physical activity at one-month follow-up. This pilot study demonstrated that the AVG program might be an effective and alternative way of exercise for people with T2D. A future RCT is warranted to determine the physical and psychological effects of AVG on T2D population.

Keywords: active video game; exergaming; type 2 diabetes; fitness; motivation

\section{Introduction}

Diabetes is an increasingly prevalent medical condition affecting 34.2 million people (10.5\% of the population) in the United States [1]. Type 2 diabetes (T2D) accounts for approximately $90-95 \%$ of all diagnosed cases of diabetes. Diabetes is marked by high blood glucose levels with hemoglobin of $\mathrm{A} 1 \mathrm{C}$ (HbA1c) $6.5 \%$ or higher. If individuals with T2D do not manage their glucose well, they may have a higher risk of developing progressive complications, such as heart disease, stroke, retinopathy, blindness, chronic kidney disease, neuropathy, amputation, and premature death [1].

Physical activity and exercise have been recommended to prevent and manage T2D [2] Engaging in regular exercise has been demonstrated to improve blood glucose control, insulin sensitivity, lipids, and quality of life as well as reducing blood pressure (BP), risk of cardiovascular disease, and mortality [3]. One meta-analysis that looked at 42 randomized control trials (RCT) found similar results, including improved BP control, decreased low-density lipoprotein cholesterol (LDL-C), and elevated high-density lipoprotein cholesterol (HDL-C) levels among people with diabetes [4]. However, individuals with diabetes are much less likely to participate in regular physical activity. In the United States, older 
adults with diabetes were reported to be 31-34\% less likely to achieve the recommended physical activity guidelines and 13-19\% less likely to achieve sufficient amounts of physical activity compared to adults without diabetes [5]. Several barriers to exercise exist among people with diabetes and have been identified through various studies. These barriers include poor health, lack of company, lack of interest, lack of time, lack of social support, cost, transportation, body image and embarrassment, access to facilities, and diabetes specific barriers, such as insufficient knowledge about managing diabetes and diabetic complications while exercising [3,6].

One emerging form of physical activity is active video gaming (AVG). AVG, or exergaming, is a video game that requires physical movement to play. In the early years, AVG involved a wireless controller with motion sensing for upper extremity (UE) movement and/or dance pads on the ground to detect lower extremity (LE) movement. Subsequent gaming systems, such as the XBOX Kinect, are camera-based and capable of tracking the entire body movement. The wide variety of games available focus on many of the components of a traditional fitness program, such as strength, balance, coordination, flexibility, agility, and aerobic endurance. AVG can be tailored to an individual's interests and preferred exercise style through numerous gaming options. It can be an individual activity, where a person seeks to improve their own personal high score, or a group activity, where multiple individuals compete against one another [7]. Therefore, AVG might be suited to reduce many of the aforementioned barriers because of the following reasons: (1) it can be performed at home, (2) individuals can play against others, (3) there is no membership cost, and (4) it is designed to be a more enjoyable mode of exercise.

Several studies have evaluated the effect of AVG as a method of exercise for children and adults. One systematic review suggested that AVG is capable of providing light-to-moderate intensity physical activity [8]. Another study found that energy expenditure, heart rate (HR), and oxygen consumption while playing AVG are significantly higher than resting values and these effect sizes were similar to traditional methods of physical exercise [9]. One study found that a healthy population that participated in a six-week AVG program showed improved flexibility, maximal oxygen consumption, and resting HR [7]. Another study found that a 12-week AVG program improved physical fitness, including diastolic BP, sit-up tests, and 3-min step tests [10]. On overweight and obese children, one RCT reported that AVG improved body mass index (BMI) and body composition [11]. However, there is limited research that focuses on the effect of AVG on people with T2D. Therefore, the objective of this pilot study was to assess the effects of an AVG program on physiological parameters, fitness levels, motivation to physical activity, and quality of life on people with T2D.

\section{Materials and Methods}

\subsection{Subjects}

Eight subjects with T2D (two males and six females; age 36 to 75 y/o with mean $55.6 \pm 13.0 \mathrm{y} / \mathrm{o}$ ) were recruited from the local community for the eight-week AVG program. The inclusion criteria were: (1) diagnosed with T2D and (2) able to understand the entire content of the consent form-including purpose of the project, procedures, potential discomfort/risks and benefits-and voluntarily consent or assent to participate in this study. The exclusion criteria were: (1) gestational diabetes (high blood sugar affecting pregnant women), (2) severe diabetic complications including stroke, heart disease, kidney failure, blindness, and amputation, (3) comorbidities or pre-existing conditions that would contraindicate exercise training, and (4) impaired mentation. The subjects reported that on average they exercised about two to three times per week (mean 2.6 times; range from zero to five times).

\subsection{Evaluation Sessions}

Initial, mid-term (at the end of the 4th week), and final evaluations were conducted using a variety of outcome measures designed to assess physiological parameters, fitness 
levels, motivation for physical activity, and quality of life. Upon arrival of the evaluation day, chest strap HR monitors (vívofit Fitness Band, Garmin Ltd., Olathe, KS, USA) were placed on subjects before having them sit to the complete health intake form (initial evaluation only) and three psychological outcome measures. Objective measures were then gathered systematically (Supplementary Materials S1) by the same researcher in subsequent evaluations. A follow-up phone interview was conducted by a researcher one month after the subjects' program completion by using a questionnaire (Supplementary Materials S2).

\subsection{Psychological Assessment}

Three outcome measures were utilized to assess motivation for physical activity and participant's quality of life. The Physical Activity Measure- Revised (MPAM-R) was used to assess the strength of five motives for participation in physical activities. The motives are fitness, appearance, competence/challenge, social, and enjoyment (Supplementary Materials S3). This scale has been validated and used to predict various behavioral outcomes such as attendance, persistence, or maintained participation in exercise activity [12]. The Situational Motivation Scale (SIMS) is a brief and versatile self-report measure used to assess situational intrinsic motivation, identified regulation, external regulation, and amotivation (Supplementary Materials S4). The construct validity of the SIMS has been supported by correlations with other constructs [13]. The World Health Organization Quality of Life (WHOQOL)-BREF was developed as a valid and reliable alternative to the WHOQOL-100 [14]. This tool was designed to serve as a brief assessment of quality of life and evaluation of treatment efficacy (Supplementary Materials S5).

\subsection{Physical and Fitness Assessment}

HR was monitored (vívofit Fitness Band, Garmin Ltd., Olathe, KS, USA) following the conclusion of paperwork and self-reported outcomes, in order to reflect resting HR as closely as possible. Maximum HR was calculated as 220 minus the subject's age. $\mathrm{HbA} 1 \mathrm{c}$ was measured using the $\mathrm{A} 1 \mathrm{CNOW}^{\circledR}$ test system (pts Diagnostics, Whitestown, IN, USA). Sedentary BP was measured in the seated position (Carescape V100 Monitor, GE Healthcare, Chicago, IL, USA). BMI was calculated from subject height and weight. The whole body composition was determined using a hand-held bioelectrical impedance analysis device (FAT LOSS monitor, Omron, Kyoto, Japan). Flexibility was measured using the Sit and Reach Test, which has been found to be a reliable and valid measure of hamstring flexibility in middle-aged to older men and women [15]. The test was repeated three times and the average was recorded in centimeters $(\mathrm{cm})$. To evaluate muscle strength, manual muscle testing was performed by the same research with the use of a hand-held dynamometer (MicroFET2 ${ }^{\circledR}$, Hoggan Scientific, LLC., Salt Lake City, UT, USA). For each muscle group, a standardized testing position (Supplementary Materials S6) was utilized and subjects were instructed to reach maximal contraction within $2 \mathrm{~s}$ and then hold until being informed to relax. Three repetitions were performed bilaterally on each muscle group and the average was calculated for each group. Muscular endurance was assessed using the One-minute Sit-Up Test. Subjects' scores were recorded as number of correct reps performed in one minute. Cardiovascular endurance was assessed using the YMCA Three-minute Step Test, which has been found to be a valid tool [16]. This outcome provides a submaximal measure of aerobic fitness based on how quickly HR returns to normal after exercise. Subjects' HR was recorded and categorized based on age-matched normative data published by YMCA [17]. If subjects were unable to complete the step test, the One-minute Sit-to-Stand test was used instead, in which subjects were instructed to rise and return to the chair (bottom must touch, but back does not have to) as many times as possible in one minute without use of upper extremities.

\subsection{Exergaming}

Exergaming sessions lasted one hour each and included five minutes of warm-up, 50 min of AVG using Xbox Kinect and/or Nintendo Wii, and five minutes of cool down (see Supplementary Materials S7 for warm-up and cool-down protocols). Prior to beginning 
warm-up, subjects were encouraged to check current blood glucose levels through finger stick (Accu-Chek ${ }^{\circledR}$ Nano, Indianapolis, IN, USA) as a precursor to before exercise. Snacks were available as needed to resolve any issues of hypoglycemia. During each session, subjects' HR was recorded in five-minute intervals using a Garmin Fitness Band reflecting data from a chest HR monitor. Subjects' perception of exercise intensity was also verbally communicated and recorded every five minutes using the Borg Rating of Perceived Exertion (RPE), which ranges from a score of 6 (no exertion) to 20 (maximum exertion) [18]. The primary games used on the Xbox included Kinect Sports and Shape Up (Figure 1). The games used on the Nintendo Wii included Just Dance, Wii Sports, and Wii Sports Resort. The subjects were able to select the games they preferred to play during each session. The subjects were asked to participate in the supervised AVG program in a central location on the university campus with the frequency of two sessions per week for eight weeks to achieve their adherence.

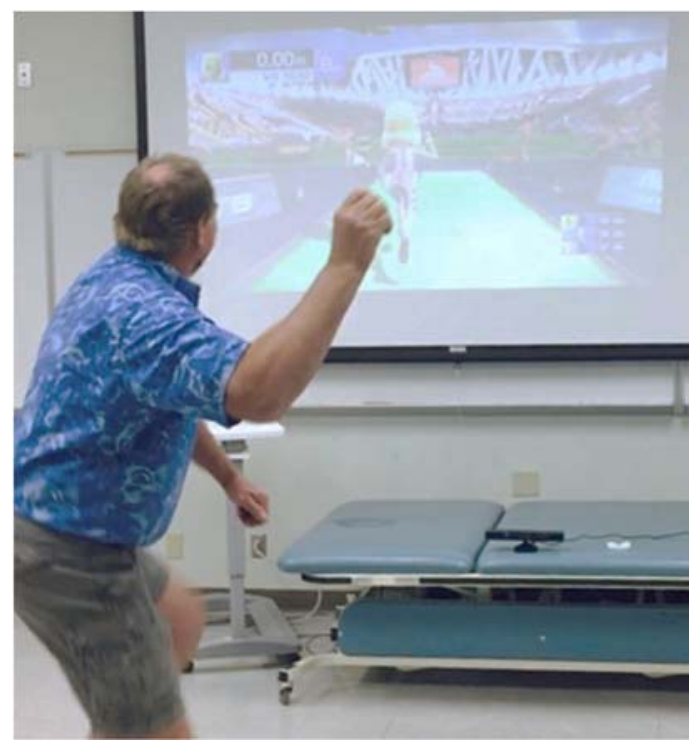

Figure 1. One subject is playing javelin throw in Kinect Sports by using Xbox Kinect.

\subsection{Statistical Analysis}

The differences in physical or psychological outcome measures between three evaluations were examined using one-way repeated measures ANOVA. Reliability of the psychological scales, the MPAM-R, SIMS, and WHOQOL-BREF was determined using Cronbach's alpha. All data were analyzed by using IBM-SPSS Statistics (version 25). Significant differences were determined by a $p$ value of $<0.05$. The data are presented as mean \pm standard deviation (SD).

\section{Results}

Eight subjects participated in the program (16 sessions for eight weeks) and one dropped out after the ninth session due to a schedule conflict. During each AVG session, on average, the subjects' HR increased $32 \pm 21 \%$ from their resting HR and peaked at $57 \pm 26 \%$ of resting HR. Subjects' exercise intensity ranged between $61 \%$ and $83 \%$ of their max $\mathrm{HR}$ and the average was $71 \pm 8 \% \max \mathrm{HR}$, indicating moderate to vigorous exercise. Subjects' average RPE throughout each session was $11.9 \pm 0.8$ and the max RPE was $14.4 \pm 0.9$.

After participating program, subjects' $\mathrm{HbA1c}$ decreased from $7.5 \%$ to $7.1 \%$. Although the changes were not statistically significant, five of seven subjects' $\mathrm{HbA} 1 \mathrm{c}$ were lower on the final evaluation when compared to initial evaluation. Systolic BP decreased from $149.1 \mathrm{mmHg}$ to $135.1 \mathrm{mmHg}$. Diastolic BP decreased from $80.8 \mathrm{mmHg}$ to $70.9 \mathrm{mmHg}$, but the changes were not statistically significant. The body weight, BMI, body fat $\%$, and HR did not have any significant change. On the muscle strength, LE flexion and extension 
increased on both left and right. For the UE, muscle strength increased on the left but decreased on the right. The flexibility increased from $19.0 \mathrm{~cm}$ to $20.7 \mathrm{~cm}$. All the changes were not statistically significant (Table 1).

Table 1. Results for Physical Assessment.

\begin{tabular}{cccc}
\hline & Initial & Mid & Final \\
\hline HbA1c $(\%)$ & $7.5 \pm 1.3$ & $7.4 \pm 1.4$ & $7.1 \pm 1.2$ \\
HR (bpm) & $90.4 \pm 12.4$ & $92.1 \pm 14.9$ & $91.0 \pm 15.8$ \\
SBP (mmHg) & $148.0 \pm 19.6$ & $135.4 \pm 16.9$ & $135.1 \pm 17.0$ \\
DBP (mmHg) & $81.4 \pm 11.3$ & $69.6 \pm 10.7$ & $70.9 \pm 14.1$ \\
Weight $(\mathrm{kg})$ & $93.6 \pm 31.4$ & $94.1 \pm 32.3$ & $95.0 \pm 33.1$ \\
BMI $\left(\mathrm{kg} / \mathrm{m}^{2}\right)$ & $33.6 \pm 8.3$ & $34.1 \pm 8.2$ & $33.7 \pm 8.1$ \\
Body Fat $(\%)$ & $40.0 \pm 6.8$ & $39.4 \pm 6.4$ & $41.3 \pm 6.9$ \\
Muscle Strength $(\mathrm{lbs})$ & & & \\
Right UE Flex & $42.3 \pm 25.3$ & $40.4 \pm 13.7$ & $41.1 \pm 15.3$ \\
Right UE Ext & $30.6 \pm 20.2$ & $24.2 \pm 6.2$ & $28.8 \pm 9.4$ \\
Right LE Flex & $33.7 \pm 17.6$ & $32.0 \pm 8.5$ & $41.3 \pm 21.1$ \\
Right LE Ext & $36.1 \pm 15.4$ & $38.8 \pm 15.4$ & $42.2 \pm 17.6$ \\
Left UE Flex & $42.0 \pm 22.6$ & $38.9 \pm 14.6$ & $31.3 \pm 11.8$ \\
Left UE Ext & $31.1 \pm 19.9$ & $28.7 \pm 14.2$ & $39.4 \pm 21.0$ \\
Left LE Flex & $31.8 \pm 18.0$ & $33.6 \pm 12.4$ & $37.0 \pm 20.8$ \\
Left LE Ext & $35.2 \pm 21.1$ & $33.6 \pm 15.4$ & $20.7 \pm 6.0$ \\
Flexibility ${ }^{1}(\mathrm{~cm})$ & $19.0 \pm 4.3$ & $21.7 \pm 5.3$ &
\end{tabular}

$\mathrm{N}=7$; Data are presented as mean \pm SD. UE Flex: elbow flexors (biceps); UE Ext: elbow extensors (triceps) LE Flex: knee flexors (hamstrings); LE Ext: knee extensors (quadriceps). ${ }^{1} \mathrm{~N}=6$. One subject could not perform the task due to limitation of body shape.

On the aerobic endurance test, five subjects participated in the YMCA Three-minute Step Test and their ratings ranged from very poor to average on the initial evaluation. Four of them completed the final test and their aerobic endurance levels remained the same. Two subjects could not participate in the step test due to physical limitations. Therefore, they completed the sit to stand test instead. The results for the aerobic endurance test are presented in Supplementary Materials S8. On the muscle endurance test, only two subjects completed all three evaluations, while the others participated in some or none of the evaluations due to lack of will, back pain, or other physical limitations (Supplementary Materials S9).

On the psychological assessment, there were no significant differences between the three evaluation sessions on MPAM-R, SIMS, and WHOQOL-BREF. The scores of the subcategories in each measurement tool and their respective internal consistency (reliability) are summarized in the Table 2.

At one-month follow-up, two participants bought an Xbox console and continued to participate in AVG after the program ended. One participant signed up for a gym membership. Six participants reported that they would continue with the program if given the opportunity. Six participants reported that they felt their attitude toward exercise had improved and five reported feeling healthier since beginning the program. 
Table 2. Results for Psychological Assessment.

\begin{tabular}{|c|c|c|c|}
\hline & Initial & Mid & Final \\
\hline \multicolumn{4}{|l|}{ MPAM-R } \\
\hline Enjoyment & $38.9 \pm 2.3$ & $35.7 \pm 10.9$ & $38.7 \pm 9.1$ \\
\hline Competence & $34.9 \pm 10.0$ & $34.9 \pm 10.1$ & $36.6 \pm 11.6$ \\
\hline Appearance & $29.6 \pm 11.2$ & $29.1 \pm 12.3$ & $29.3 \pm 13.1$ \\
\hline Fitness & $29.7 \pm 6.0$ & $31.6 \pm 2.4$ & $30.9 \pm 4.4$ \\
\hline Social & $23.7 \pm 7.6$ & $15.7 \pm 9.1$ & $18.9 \pm 8.9$ \\
\hline Cronbach's Alpha & 0.887 & 0.955 & 0.967 \\
\hline (Internal Consistency) & (Good) & (Excellent) & (Excellent) \\
\hline \multicolumn{4}{|l|}{ SIMS } \\
\hline Intrinsic Motivation & $22.9 \pm 3.8$ & $22.6 \pm 4.0$ & $22.9 \pm 4.7$ \\
\hline Identified Regulation & $25.3 \pm 4.2$ & $24.7 \pm 3.3$ & $26.0 \pm 2.2$ \\
\hline External Regulation & $9.7 \pm 4.5$ & $10.1 \pm 7.5$ & $9.6 \pm 4.6$ \\
\hline Amotivation & $8.3 \pm 2.9$ & $7.0 \pm 4.6$ & $7.6 \pm 4.3$ \\
\hline Cronbach's Alpha & 0.686 & 0.739 & 0.567 \\
\hline (Internal Consistency) & (Questionable) & (Acceptable) & (Poor) \\
\hline \multicolumn{4}{|l|}{ WHOQOL-BREF } \\
\hline Physical & $13.6 \pm 1.4$ & $12.2 \pm 1.6$ & $13.1 \pm 1.7$ \\
\hline Psychological & $14.0 \pm 2.0$ & $13.5 \pm 1.1$ & $14.0 \pm 1.6$ \\
\hline Social Relationships & $15.4 \pm 2.9$ & $15.8 \pm 2.6$ & $15.6 \pm 1.8$ \\
\hline Environment & $16.2 \pm 2.7$ & $15.7 \pm 2.2$ & $16.3 \pm 1.8$ \\
\hline Cronbach's Alpha & 0.863 & 0.893 & 0.600 \\
\hline (Internal Consistency) & (Good) & (Excellent) & (Questionable) \\
\hline
\end{tabular}

$\mathrm{N}=7$; Data are presented as mean $\pm \mathrm{SD}$.

\section{Discussion}

The American Diabetes Association recommends people with diabetes should engage in at least $150 \mathrm{~min} /$ week moderate to vigorous-intensity exercise [2]. Recently, Höchsmann et al. reported that T2D patients were able to achieve moderate exercise during AVG with Wii Fit Plus [19]. In the present study, AVG using Xbox Kinect and/or Wii also generated moderate intensity exercise based on percentage of max HR. Some participants at times were even able to reach vigorous levels. The results of max RPE indicated between somewhat hard and hard on the scale [20]. As to the duration, the subjects in this study participated in 120-180 min of AVG exercises per week. Therefore, an AVG program may be a feasible alternative exercise training that provides equivalent intensity and duration for people of T2D to reach the established exercise recommendation.

The effect of games on blood glucose levels $(\mathrm{HbA} 1 \mathrm{c})$ for people with diabetes has been accessed recently and their impact has been suggested to be low [21]. Kempf and Martin conducted an RCT and provided T2D patients with a Wii Fit Plus sports games to use at home for $30 \mathrm{~min} /$ day for 12 weeks. They found the intervention group significantly improved $\mathrm{HbA} 1 \mathrm{c}$ from $7.1 \%$ to $6.8 \%$ (0.3\% decrease) [22]. In this study, the T2D patients participated in a supervised AVG program in a central location for $60 \mathrm{~min} / \mathrm{session}$, two to three sessions/week for eight weeks. Their $\mathrm{HbA} 1 \mathrm{c}$ decreased from $7.5 \%$ to $7.1 \%(0.4 \%$ decrease), despite the variation on background characteristic between subjects was wide, which is a limitation of this study. Although the decrease was not statistically significant due to the sample size, five out of seven participants showed an improvement on $\mathrm{HbA1c}$ levels in only eight weeks. Therefore, playing exercise games at home or within structured settings (e.g., gyms or clinics) might be beneficial for blood glucose management on people with T2D. Another positive trend noted was the increase in LE muscle strength across the participants, even though the results were not statistically significant. The reason might be due to playing AVG requires standing and involves a significant amount of leg movement. In the same manner, although not statistically significant, the body flexibility was increased. This might be due to the various physical movements in the games, including kicking and punching in multiple directions, squats, lunges, jogging in place, and dancing. 
There were a few participants not able to complete some of the fitness assessment. One participant was unable to sit on the floor for the sit and reach flexibility test. Three participants were unable to complete the YMCA Three-minute Step Test, which seemed to be physically too challenging for them. Two participants refused to participate in the sit up test due to pain. Another participant showed the attempt but failed to complete one full sit up. The challenge of sit up tests in this study was similar to the difficulties reported in another study on people with diabetes [23]. Therefore, for people with certain of physical limitations, a modified or alternative test based on the individual's physical capability should be considered. For instance, the functional reach test might be a better option of flexibility test for people with obesity, because it consists of having the subject in a standing position and measuring how far they can reach past arm's length [23]. For the aerobic endurance test, the One-minute Sit to Stand Test might be more physically doable for older adults instead of the Three-minute Step Test.

The MPAM-R was used to assess participant's motivation and projected exercise adherence. The reliability has been reported in healthy adults [12], healthy children [24], and overweight and obese children [25]. In the present study, the MPAM-R revealed a high internal consistency on T2D population, even though the changes in each category were not significant between evaluations. The SIMS was selected to assess participant's intrinsic and extrinsic motivation as a reliable instrument [26]. The results here on T2D population did not produce scores with acceptable reliability, which is similar to the outcome in another AVG study on overweight and obese children [25]. Recently, WHOQOL-BREF has been suggested to be reliable for clinical and research uses on T2D patients [27]. Here, the results showed good to excellent internal consistency except for the final evaluation on T2D patients who participated in an AVG program. Thus, the WHOQOL-BREF may have some questionable test-retest reliability when using it across several sessions. In addition, the differences from the beginning and to the end in each category were not significant. It is important to note that scores on motivation and quality of life assessment were high during the initial evaluation, which may indicate that there might be a ceiling effect. Therefore, there was little to no room for a significant positive change. Although changes on psychological assessment were not significant, the participants overall revealed positive mood trends throughout the AVG program. At one-month follow-up via phone interviews, six of seven participants reported better behavior and attitudes toward their health and physical activity. Two participants carried on playing AVG at home while one participant joined a gym. On average, the participants continued to exercise for about $110 \mathrm{~min}$ per week.

\section{Conclusions}

This pilot study demonstrated that AVG could be an alternative form of moderate intensity exercise for people with T2D. An eight-week supervised AVG program improved $\mathrm{HbA1c}, \mathrm{BP}$, and LE muscle strength, even though the results were not statistically significant due to sample size. One-month follow-up showed participating in the AVG program might have positive impact on T2D, patient's behavior, and attitudes toward health and physical activity. A future RCT with a larger sample size and more physically-achievable tests for fitness assessment is warranted to determine the effect of AVG on the T2D population.

Supplementary Materials: The following are available online at https://www.mdpi.com/article/10 .3390/app112211046/s1, Supplementary Materials S1: Order of Evaluation Procedures. Supplementary Materials S2: Follow-Up Questionnaire. Supplementary Materials S3: Motivates for Physical Activities Measure- Revised (MPAM-R). Supplementary Materials S4: Situational Motivation Scale (SIMS). Supplementary Materials S5: World Health Organization Quality of Life (WHOQOL). Supplementary Materials S6: Muscle Strength Test Positioning. Supplementary Materials S7: Warm-Up and Cool-Down Procedures. Supplementary Materials S8: Results for Aerobic Endurance Test. Supplementary Materials S9: Results for Muscular Endurance Test.

Author Contributions: Conceptualization, H.-H.H., B.G., R.H., H.I. and E.S.; methodology, H.-H.H., B.G., R.H., H.I. and E.S..; validation, H.-H.H. and D.A.C.; formal analysis, H.-H.H. and D.A.C.; investigation, B.G., R.H., H.I. and E.S.; resources, H.-H.H.; data curation, B.G., R.H., H.I. and E.S.; 
writing-original draft preparation, B.G., R.H., H.I. and E.S.; writing-review and editing, H.-H.H. and D.A.C.; supervision, H.-H.H.; project administration, H.-H.H. All authors have read and agreed to the published version of the manuscript.

Funding: This research received no external funding.

Institutional Review Board Statement: The study was conducted according to the guidelines of the Declaration of Helsinki, and approved by the Institutional Review Board of Angelo State University (HUA-030116).

Informed Consent Statement: Informed consent was obtained from all subjects involved in the study. Written informed consent has been obtained from the patient(s) to publish this paper.

Data Availability Statement: Data is contained within the article and Supplementary Material.

Acknowledgments: The authors would like to acknowledge the World Health Organization (WHO) for authorization to reprint the WHOQOL-BREF as appendix for this manuscript.

Conflicts of Interest: The authors declare no conflict of interest.

\section{References}

1. U.S. Dept of Health and Human Services. Centers for Disease Control and Prevention. In National Diabetes Statistics Report; U.S. Dept of Health and Human Services: Washington, DC, USA, 2020.

2. American Diabetes Association. 5. Facilitating Behavior Change and Well-being to Improve Health Outcomes: Standards of Medical Care in Diabetes-2020. Diabetes Care 2020, 43 (Suppl. S36), S48-S65. [CrossRef]

3. Qiu, S.H.; Sun, Z.L.; Cai, X.; Liu, L.; Yang, B. Improving patients' adherence to physical activity in diabetes mellitus: A review. Diabetes Metab. J. 2012, 36, 1-5. [CrossRef]

4. Hayashino, Y.; Jackson, J.L.; Fukumori, N.; Nakamura, F.; Fukuhara, S. Effects of supervised exercise on lipid profiles and blood pressure control in people with type 2 diabetes mellitus: A meta-analysis of randomized controlled trials. Diabetes Res. Clin. Pract. 2012, 98, 349-360. [CrossRef]

5. Zhao, G.; Ford, E.S.; Li, C.; Balluz, L.S. Physical activity in U.S. older adults with diabetes mellitus: Prevalence and correlates of meeting physical activity recommendations. J. Am. Geriatr. Soc. 2011, 59, 132-137. [CrossRef]

6. Lascar, N.; Kennedy, A.; Hancock, B.; Jenkins, D.; Andrews, R.C.; Greenfield, S.; Narendran, P. Attitudes and barriers to exercise in adults with type 1 diabetes (T1DM) and how best to address them: A qualitative study. PLoS ONE 2014, 9, e108019. [CrossRef]

7. Roopchand-Martin, S.; Nelson, G.; Gordon, C.; Sing, S.Y. A pilot study using the XBOX Kinect for exercise conditioning in sedentary female university students. Technol. Health Care Off. J. Eur. Soc. Eng. Med. 2015, 23, 275-283. [CrossRef]

8. Peng, W.; Crouse, J.C.; Lin, J.H. Using active video games for physical activity promotion: A systematic review of the current state of research. Health Educ. Behav. Off. Publ. Soc. Public Health Educ. 2013, 40, 171-192. [CrossRef]

9. Peng, W.; Lin, J.H.; Crouse, J. Is playing exergames really exercising? A meta-analysis of energy expenditure in active video games. Cyberpsychology Behav. Soc. Netw. 2011, 14, 681-688. [CrossRef]

10. Huang, H.-C.; Wong, M.-K.; Lu, J.; Huang, W.-F.; Teng, C.I. Can using exergames improve physical fitness? A 12-week randomized controlled trial. Comput. Hum. Behav. 2017, 70, 310-316. [CrossRef]

11. Maddison, R.; Foley, L.; Ni Mhurchu, C.; Jiang, Y.; Jull, A.; Prapavessis, H.; Hohepa, M.; Rodgers, A. Effects of active video games on body composition: A randomized controlled trial. Am. J. Clin. Nutr. 2011, 94, 156-163. [CrossRef]

12. Ryan, R.M.; Frederick, C.M.; Lepes, D.; Rubio, N.; Sheldon, K.M. Intrinsic motivation and exercise adherence. Int. J. Sport Psychol. 1997, 28, 335-354.

13. Guay, F.; Vallerand, R.J.; Blanchard, C. On the Assessment of Situational Intrinsic and Extrinsic Motivation: The Situational Motivation Scale (SIMS). Motiv. Emot. 2000, 24, 175-213. [CrossRef]

14. The WHOQOL Group. Development of the World Health Organization WHOQOL-BREF quality of life assessment. Psychol. Med. 1998, 28, 551-558. [CrossRef]

15. Lemmink, K.A.; Kemper, H.C.; de Greef, M.H.; Rispens, P.; Stevens, M. The validity of the sit-and-reach test and the modified sit-and-reach test in middle-aged to older men and women. Res. Q. Exerc. Sport 2003, 74, 331-336. [CrossRef] [PubMed]

16. Kieu, N.T.V.; Jung, S.J.; Shin, S.W.; Jung, H.W.; Jung, E.S.; Won, Y.H.; Kim, Y.G.; Chae, S.W. The Validity of the YMCA 3-Minute Step Test for Estimating Maximal Oxygen Uptake in Healthy Korean and Vietnamese Adults. J. Lifestyle Med. 2020, 10, 21-29. [CrossRef] [PubMed]

17. Golding, L.A.; Myers, C.R.; Sinning, W.E. Y's Way to Physical Fitness: The Complete Guide to Fitness Testing and Instruction, 3rd ed.; YMCA of the USA by Human Kinetics Publishers: Champaign, IL, USA, 1989.

18. Scherr, J.; Wolfarth, B.; Christle, J.W.; Pressler, A.; Wagenpfeil, S.; Halle, M. Associations between Borg's rating of perceived exertion and physiological measures of exercise intensity. Eur. J. Appl. Physiol. 2013, 113, 147-155. [CrossRef]

19. Höchsmann, C.; Zürcher, N.; Stamm, A.; Schmidt-Trucksäss, A. Cardiorespiratory Exertion While Playing Video Game Exercises in Elderly Individuals With Type 2 Diabetes. Clin. J. Sport Med. Off. J. Can. Acad. Sport Med. 2016, 26, 326-331. [CrossRef] [PubMed] 
20. Roy, B.A. Monitoring Your Exercise Intensity. ACSM's Health Fit. J. 2015, 19, 3-4. [CrossRef]

21. Martos-Cabrera, M.B.; Membrive-Jiménez, M.J.; Suleiman-Martos, N.; Mota-Romero, E.; Cañadas-De la Fuente, G.A.; GómezUrquiza, J.L.; Albendín-García, L. Games and Health Education for Diabetes Control: A Systematic Review with Meta-Analysis. Healthcare 2020, 8, 399. [CrossRef]

22. Kempf, K.; Martin, S. Autonomous exercise game use improves metabolic control and quality of life in type 2 diabetes patients-A randomized controlled trial. BMC Endocr. Disord. 2013, 13, 57. [CrossRef]

23. Cimbiz, A.; Cakir, O. Evaluation of balance and physical fitness in diabetic neuropathic patients. J. Diabetes Its Complicat. 2005, 19, 160-164. [CrossRef] [PubMed]

24. Dishman, R.K.; Saunders, R.P.; McIver, K.L.; Dowda, M.; Pate, R.R. Construct validity of selected measures of physical activity beliefs and motives in fifth and sixth grade boys and girls. J. Pediatric Psychol. 2013, 38, 563-576. [CrossRef] [PubMed]

25. Huang, H.-H.; Cook, L.; Harrison, J.; O'Keefe, N.; Schultz, K.; Curtis, D.A. Exercise by playing active video gaming in overweight and obese children. J. Phys. Ther. Sports Med. 2017, 1, 25-31. [CrossRef]

26. Standage, M.; Duda, J.L.; Treasure, D.C.; Prusak, K.A. Validity, Reliability, and Invariance of the Situational Motivation Scale (SIMS) Across Diverse Physical Activity Contexts. J. Sport Exerc. Psychol. 2003, 25, 19-43. [CrossRef]

27. Abbasi-Ghahramanloo, A.; Soltani-Kermanshahi, M.; Mansori, K.; Khazaei-Pool, M.; Sohrabi, M.; Baradaran, H.R.; Talebloo, Z.; Gholami, A. Comparison of SF-36 and WHOQoL-BREF in Measuring Quality of Life in Patients with Type 2 Diabetes. Int. J. Gen. Med. 2020, 13, 497-506. [CrossRef] 